\title{
Avaliação das Relações Familiares por Idosos com Diferentes Condições Sociodemograficas e de Saúde
}

\author{
Dóris Firmino Rabelo - Universidade Federal do Recôncavo da Babia, Santo Antônio de Jesus, Brasil \\ Anita Liberalesso Neri - Universidade Estadual de Campinas, Campinas, Brasil
}

\begin{abstract}
Resumo
O objetivo deste estudo foi investigar relações entre avaliações do funcionamento familiar feitas por idosos e suas condições sociodemograficas e de saúde. Participaram 134 idosos, submetidos a medidas de autorrelato sobre a satisfação com as relações familiares e o clima familiar, sexo, idade e configuração familiar. Os dados passaram por análise de conglomerados. Resultaram três agrupamentos para cuja diferenciação mais contribuíram as variáveis independência funcional $\left(\mathrm{R}^{2}=0,816\right)$ e satisfação com as relações familiares $\left(\mathrm{R}^{2}=0,642\right)$. Foram observadas relações entre independência funcional, ausência de depressão e ansiedade, satisfação com as relações familiares e avaliação positiva do clima familiar; depressão e ansiedade, insatisfação com as relações familiares e avaliação negativa do clima familiar; dependência funcional, depressão e ansiedade, satisfação com as relações familiares e avaliação negativa do clima familiar, sugerindo que dependência e distúrbios emocionais podem funcionar como condições de risco à funcionalidade familiar.

Palavras-chave: relações familiares, saúde da família, idosos, família
\end{abstract}

\section{Evaluation of Family Relationships by Aged People with Different Health and Sociodemographic Conditions}

\begin{abstract}
This study was aimed to investigate associations between the evaluations of family relationships performed by elderly people and their sociodemographic and health characteristics. 134 seniors were submitted to self-report measures of satisfaction with family relationships and family climate, sex, age and family configuration. Data were submitted to cluster analysis from which there were derived three variable groups whose differentiation was mostly determined by functional independence $\left(R^{2}=0.816\right)$ and satisfaction with family relationships $\left(\mathrm{R}^{2}=0.642\right)$. There we observed statistically significant associations between functional independence, absence of depression and anxiety, satisfaction with family relationships, and positive evaluation towards family climate; depression and anxiety, dissatisfaction with family relationships, and negative evaluation of the family climate; functional dependence, depression and anxiety, satisfaction with family relationships and negative evaluation of the family climate, suggesting that functional dependence and emotional disturbances can be risk conditions towards family functionality. Keywords: family relationships, family health, elderly, family
\end{abstract}

\section{Evaluación de las Relaciones Familiares por Personas Mayores con Diferentes Condiciones Sociodemográficas y de Salud}

\begin{abstract}
Resumen
El objetivo de este estudio fue investigar relaciones entre evaluaciones del funcionamiento familiar realizadas por personas mayores y sus condiciones sociodemográficas y de salud. Participaron 134 personas ancianas que fueron sometidas a medidas de autoinforme sobre la satisfacción con las relaciones familiares y el clima familiar, sexo, edad y configuración familiar. Los datos pasaron por análisis de conglomerados, resultando tres grupos, cuya diferenciación se determinó principalmente por la variable independencia funcional $\left(R^{2}=0,816\right)$ y satisfacción con las relaciones familiares $\left(R^{2}=0.642\right)$. Fueron observadas relaciones entre independencia funcional, ausencia de depresión y ansiedad, satisfacción con las relaciones familiares y evaluación positiva del clima familiar; depresión y ansiedad, insatisfacción con las relaciones familiares y evaluación negativa del clima familiar; dependencia funcional, depresión y ansiedad, satisfacción con las relaciones familiares y evaluación negativa del clima familiar; lo que sugiere que dependencia y trastornos emocionales pueden funcionar como condiciones de riesgo hacia la funcionalidad familiar.

Palabras-clave: relaciones familiares, salud de la família, ancianos, familia
\end{abstract}

\section{Introdução}

Cada fase do ciclo vital é caracterizada por desafios específicos, que determinam as formas de ser e estar, as forças e as vulnerabilidades dos indivíduos e de suas famílias. Para os idosos e para as famílias com idosos, os principais desafios a serem enfrentados são decorrentes do declínio fisiológico normativo do envelhecimento, das limitações e dos riscos que afetam a saúde dos mais velhos, das mudanças em papéis sociais decorrentes do relativo afastamento dos idosos da vida social, das alterações nas relações entre as gerações e das alterações nas configurações e na funcionalidade familiar decorrentes desses processos em interação. Deles 
emergem novas funções, regras e papeis sociais que se espelham em novas formas de expressão e de qualificação das relações interpessoais. As condições de saúde física e psicológica e de participação social dos idosos e a configuração e o funcionamento de suas famílias formam sistemas relacionais e emocionais complexos (Piercy, 2010) de grande importância para a adaptação dos idosos.

A percepção dos idosos sobre o funcionamento familiar diz respeito à avaliação que fazem sobre a qualidade das relações, sobre o grau da eficácia exibida pela família no atendimento de suas necessidades e sobre o grau em que as relações familiares correspondem às suas expectativas. A satisfação dos idosos com relação ao funcionamento da família associa-se de forma robusta com a percepção de coesão e de conforto emocional que derivam de suas relações com pessoas que lhes são significativas, nesse contexto (Fingerman \& Birditt, 2011; Lowenstein, 2007). A percepção de que há pessoas disponíveis para ajudar no dia a dia e em tempos de crise e de necessidades especiais, atua como moderador do efeito dos eventos estressantes sobre o bem-estar psicológico dos idosos (Hyde, Gorka, Manuck, \& Hariri, 2011) e afeta positivamente a sua saúde física e mental (Batistoni et al., 2013). Na velhice, o principal motivo para as trocas sociais é a ampliação dos sensos de coesão e de proximidade emocional (Lang, Wagner, Wrzus, \& Neyer, 2013).

A satisfação dos idosos com as suas relações familiares é um indicador importante do funcionamento familiar (Rodríguez-Sánchez et al., 2011), expresso nos atributos coesão, apoio, conflito e hierarquia (Teodoro, Allgayer \& Land, 2009). A hierarquia é um requisito fundamental para o funcionamento da família, para o cumprimento dos papeis que lhe são atribuídos e para a sua capacidade de adaptação. Alterações na estrutura de poder e controle, habitualmente vigentes numa estrutura familiar, podem gerar tensão nos relacionamentos. O conflito é um aspecto normativo das relações familiares, mas também pode determinar aumento da ansiedade dos membros. Quando intenso, pode indicar dificuldade no equilíbrio entre necessidades individuais e as da unidade familiar. Em associação com o significado que a hierarquia tem para seus integrantes, os níveis de coesão, apoio e conflito da família determinam a qualidade percebida das relações intergeracionais, bem como as consequências dessa qualidade sobre a funcionalidade da família e sobre o bem-estar de seus membros (Fingerman \& Birditt, 2011; Lowenstein, 2007).
A maioria dos idosos relata satisfação com seus relacionamentos familiares (Paiva et al., 2011; Pavarini et al., 2006; Santos, Pavarini, \& Barham, 2011; Silva, Rabelo, \& Queroz, 2010). Mulheres idosas, idosos que moram sozinhos ou em arranjos mistos (familiares próximos e distantes e/ou empregados e/ou amigos) e aqueles que não têm expectativa de virem a ser cuidados por um familiar em caso de doença, geralmente pontuam mais baixo em escalas de satisfação do que homens idosos, idosos que vivem em arranjos conjugais e os que têm expectativa positiva de cuidado.

Em famílias com idosos funcionalmente dependentes são observadas taxas mais altas de disfunção familiar do que em famílias sem idosos dependentes, sugerindo que a incapacidade dos idosos é um estressor cuja intensidade supera os recursos de enfrentamento da família (Torres, Reis, Reis, \& Fernandes, 2009; Torres, Reis, Reis, Fernandes \& Xavier, 2010). Idosos com limitações funcionais expressivas têm maior risco de desenvolver depressão e ansiedade (Vink, Aartsen, \& Schoevers, 2008) e de viver em arranjos domiciliares não continentes do que idosos independentes (Camarano \& Kanso, 2010). A depressão e a ansiedade refletem-se em desregulação emocional e tendem a afetar negativamente a percepção do suporte social disponível e do funcionamento familiar (Carl, Soskin, Kerns, \& Barlow, 2013; Werner-Seidler, Banks, Dunn, \& Moulds, 2012).

A convivência com membros idosos doentes, com incapacidades físicas ou com saúde psicológica prejudicada propõe à família um conjunto de desafios com forte potencial para, por um lado, dar origem a conflitos; por outro, a novas formas de funcionamento e a novas configurações (por exemplo, coabitação entre membros idosos e não idosos). Lidar de forma eficaz com essas condições envolve o concurso de recursos que favoreçam a harmonia, a funcionalidade e a estabilidade familiar (Torres et al., 2010; Santos, Pavarini, \& Barham, 2011). Ou seja, os desafios gerados pela presença de idosos doentes e incapacitados podem ser um veículo positivo de mudança, de reestruturação e de fortalecimento dos vínculos familiares (Resende et al., 2008). A percepção dos membros idosos e não idosos da família de que as necessidades individuais e coletivas são respondidas é um indicador de funcionamento familiar saudável (Silverstein \& Giarrusso, 2010).

O objetivo deste estudo é identificar relações entre as avaliações realizadas por idosos com respeito ao funcionamento de suas famílias, as condições de 
saúde física e psicológica desses idosos e suas características sociodemográficas.

\section{Método}

\section{Local e Participantes}

O campo selecionado para estudo foi Santo Antonio de Jesus, Estado da Bahia - Brasil, cujo papel no setor da saúde é importante no Recôncavo Baiano. Sede da $4^{a}$. Diretoria Regional de Saúde, a cidade nucleia a microrregião leste do estado. Dentre as 20 unidades básicas de saúde (UBS) localizadas na zona urbana do município, foi selecionada a que tinha o maior número de idosos cadastrados, correspondente a 16,8\% dos idosos residentes na cidade, e a $14,5 \%$ do total de pessoas cadastradas no sistema de saúde. Segundo dados do Sistema de Informação da Atenção Básica, ano 2010, nessa unidade estavam cadastradas 2.754 famílias que somavam 9.234 pessoas, dentre as quais 1344 tinham 60 anos e mais.

O tamanho da amostra foi fixado conforme um nível de confiança de $90 \%$, para um erro amostral de 5\%. Participaram do estudo 134 idosos, com idade entre 60 e 95 anos ( $M=72 \pm 8$ anos), a maioria do sexo feminino (77,6\%), afrodescendentes $(29,9 \%$ negros e $38,8 \%$ pardos), com renda familiar de até dois salários mínimos $(75,4 \%)$, aposentados $(75,4 \%)$ ou pensionista $(14,9 \%)$ e proprietários da residência $(79,9 \%)$.

Com a ajuda de agentes comunitários de saúde, foram realizados a identificação e o arrolamento dos domicílios familiares da UBS selecionada que contavam com um ou mais membros idosos em sua composição. Os critérios de elegibilidade adotados por ocasião do recrutamento foram: idade igual ou superior a 60 anos; residência permanente na região e no domicílio; compreensão das instruções; interesse em participar e assinatura do Termo de Consentimento Livre e Esclarecido.

Os critérios de exclusão foram: déficit auditivo ou visual grave; dificuldades de expressão verbal e de compreensão, estar temporária ou permanentemente acamado e déficit cognitivo sugestivo de demência. Para os três primeiros critérios, foram levados em conta os relatos dos próprios idosos ou de seus familiares e a observação dos recrutadores. Para o quarto critério de exclusão, foi considerada a obtenção de pontuação inferior à nota de corte num teste de rastreio cognitivo para idosos. Trata-se do Mini-Exame do Estado Mental (MEEM), instrumento de aplicação simples e rápida, com 30 itens que avaliam orientação espacial e temporal, memória imediata e atrasada, nomeação, compreensão, atenção, cálculo e praxia visuoconstrutiva. A pontuação de cada idoso é ajustada pelo valor correspondente ao número de anos de escolaridade formal que cumpriu. Nesta pesquisa, foram adotados os pontos de corte estabelecidos por Brucki, Nitrini, Caramelli, Bertolucci e Okamoto (2003), menos um desvio padrão.

A opção pela exclusão dos idosos com pontuação no MEEM sugestiva de déficit cognitivo compatível com demência foi feita para não prejudicar a confiabilidade das respostas de autorrelato dos idosos (Neri et al., 2013). Em cada domicílio, todos os idosos que se dispuseram a participar da situação de recrutamento foram entrevistados com vistas a virem a integrar a amostra. Em cada domicílio, foi escolhido o participante que obteve a maior pontuação no MEEM.

\section{Variáveis e Instrumentos}

Foi elaborado um questionário para colher dados sobre as características sociodemográficas e as da configuração familiar dos idosos: idade (agrupada em duas faixas - 60 a 74 anos e 75 anos ou mais), sexo (feminino x masculino), chefia familiar ( $\operatorname{sim}$ x não), contribuição financeira do idoso para o sustento da família (total, parcial ou nenhuma) e arranjo de moradia (sozinho, com o cônjuge ou companheiro, com o cônjuge e descendentes, com descendentes e outros tipos de arranjo).

A investigação sobre as condições de saúde física autorrelatadas incluiu, em primeiro lugar, nove itens dicotômicos sobre doenças crônicas de maior prevalência entre idosos (ex.: hipertensão e diabetes). Em cada um era perguntado se, nos 12 meses anteriores à entrevista, algum médico havia dito que o participante tinha ou não a moléstia. Nove itens dicotômicos sobre sinais e sintomas vivenciados nos últimos 12 meses (ex.: problemas de memória, distúrbios de sono e quedas) receberam o mesmo tratamento. Para ambas as medidas foram criadas as faixas nenhum(a), 1 ou 2 e 3 ou mais (Neri \& Guariento, 2011). Em segundo lugar, nesse módulo, foram realizadas medidas de autorrelato sobre o grau de independência para a realização de atividades instrumentais (Santos \& Virtuoso-Junior, 2008) e básicas de vida diária (Lino, Pereira, Camacho, Ribeiro, \& Buksman, 2008). Entre as primeiras foram avaliadas: telefonar, usar transportes, fazer compras, cozinhar, realizar serviços domésticos, fazer uso de medicação e manejo de dinheiro. Entre as segundas: banho, vestir-se, toalete, transferência, controle esfincteriano e alimentação. Ambas as escalas 
permitiam a categorização dos participantes como totalmente independentes, parcialmente dependentes ou totalmente dependentes. Ainda em condições de saúde física, foi avaliado o grau de envolvimento social, indicado pela participação em atividades físicas em situação de lazer e em atividades sociais em centro de convivência, na comunidade e em igrejas e templos. Foi adotada como nota de corte a mediana relativa ao número de atividades dessa natureza desempenhadas pelos idosos.

As condições de saúde psicológica foram avaliadas pela Escala de Depressão Geriátrica (EDG - Almeida \& Almeida, 1999) e pelo Inventário de Ansiedade de Beck - BAI (Cunha, 2001). A primeira contém 15 itens dicotômicos e é usada para rastreio de sintomas depressivos nos últimos sete dias em idosos (ex.: humores disfóricos, sensos de inferioridade e de inutilidade). Tem notas de corte $\geq 6$, considerada sugestiva de depressão leve e $\geq 11$ indicada como sugestiva de depressão grave. A segunda é composta por 21 itens sobre sintomas comuns de ansiedade na última semana. Cada item apresenta quatro possibilidades de resposta. A nota de corte para ansiedade leve é $\geq 11$; para ansiedade moderada, $\geq 20$ e para ansiedade grave, $\geq 31$, numa pontuação que pode variar entre 1 e 63 pontos. $\mathrm{Na}$ análise de dados, as pontuações $\geq 6$ para depressão e $\geq 11$ para a ansiedade) foram consideradas como sim, e as pontuação $\leq 5$ para depressão e $\leq 10$ para ansiedade, como não.

O funcionamento familiar foi avaliado mediante dois instrumentos: o APGAR da Família (Smilkstein, 1978) e o Inventário do Clima Familiar - ICF (Teodoro, Allgayer, \& Land, 2009). O primeiro contém cinco questões avaliadas de 0 (nunca), 1 (algumas vezes) ou 2 (sempre) sobre a satisfação com as relações familiares em cinco domínios: adaptação, companheirismo, desenvolvimento, afetividade e capacidade resolutiva (daí o acrônimo APGAR). O escore de 0 a 4 indica elevada disfunção familiar, de 5 a 6 indica moderada disfunção familiar e de 7 a 10 indica boa funcionalidade familiar. Posteriormente, as pontuações elevada e moderada foram consideradas como disfunção familiar, que foi contrastada com boa funcionalidade familiar. A medida de clima familiar engloba os domínios apoio, coesão, conflito e hierarquia, cada um dos quais correspondentes a um numero determinado de itens. Foi calculada a mediana da distribuição dos valores obtidos pelos idosos em cada domínio e construídos os indicadores maior e menor apoio, coesão, conflito e hierarquia.

\section{Procedimentos}

A coleta de dados foi realizada nos domicílios. Por ocasião do recrutamento, os idosos selecionados eram informados sobre o dia e o horário em que os entrevistadores voltariam para a coleta de dados. As entrevistas foram realizadas em duas ou três visitas com duração média de duas horas cada. Os idosos foram informados sobre os objetivos e o conteúdo da pesquisa, a garantia de sigilo, o caráter voluntário da participação e a possibilidade de livremente abandonar a entrevista a qualquer momento. Também eram esclarecidos sobre a ausência de riscos potenciais de natureza física ou psicológica aos participantes da pesquisa. Todos os idosos assinaram um termo de consentimento livre e esclarecido, redigido de acordo com a Resolução nº 196/96 do Conselho Nacional de Saúde (CNS), do Ministério da Saúde do Brasil, o qual compunha o projeto da pesquisa "Desenvolvimento familiar e o idoso: rede de suporte social, dinâmica familiar e a convivência intergeracional", aprovado pelo Comitê de Ética em Pesquisa da Faculdade Maria Milza (FAMAM), do município de Cruz das Almas - Bahia

Os dados foram submetidos à analise estatística descritiva. Com o objetivo de estudar o perfil da amostra, foi feita análise de conglomerados mediante o método de partição, no qual o número de clusters é estabelecido previamente (Pereira, 1999). Trata-se de análise multivariada baseada nas distribuições de frequência de um conjunto de variáveis categóricas. Não indica nem correlação e nem o peso relativo de certas variáveis sobre outra. É útil para indicar as tendências gerais da distribuição conjunta das variáveis de interesse. Os agrupamentos são comparados com a teoria e com dados de pesquisa. Com base nessas comparações são interpretados.

As variáveis foram previamente padronizadas (valor de cada uma menos a média dividido pelo desvio padrão), de forma que se pode dizer que todas tinham a mesma magnitude, evitando-se que a diferença de escala das variáveis originais pesasse na formação dos conglomerados. Mesmo as variáveis categóricas foram transformadas, pois foram atribuídos valores numéricos ordinais para as categorias.

Inicialmente, foi tentada uma solução com dois conglomerados e, em seguida, com três. Esta resultou em arranjos melhores, caracterizados por $\mathrm{R}^{2}$ mais altos e com maior número de variáveis. A forma usada para aferir a qualidade da solução encontrada foi avaliar se havia possibilidade de discriminar entre os 
conglomerados com base nas variáveis que os compunham (Kaufman \& Rousseeuw, 1990).

Com base nos testes Qui-quadrado e Exato de Fisher (nível de significância de 5\%), foram feitas comparações entre as proporções de respostas às categorias das variáveis que compuseram os conglomerados.

\section{Resultados}

$\mathrm{Na}$ análise de conglomerados a formação com três grupos correspondeu a um $\mathrm{R}^{2}$ de 0,221 , ou seja, explicou $22,1 \%$ da variabilidade dos dados. As variáveis que mais contribuíram para a formação dos conglomerados foram o desempenho de atividades básicas de vida diária $\left(\mathrm{R}^{2}=0,816\right)$ e a funcionalidade familiar indicada pelo escore geral da satisfação com os relacionamentos familiares $\left(R^{2}=0,642\right)$ (Tabelas 1 e 2$)$.

A composição dos conglomerados foi a seguinte:

Conglomerado 1 ( $n=4 ; 3 \%$ da amostra): formado por idosos com dependência total ou parcial em atividades básicas e dependência total em atividades instrumentais de vida diária, com depressão e ansiedade, satisfeitos com a adaptação, o companheirismo, o desenvolvimento e a capacidade resolutiva da família, que avaliaram como boa a funcionalidade familiar, e que relataram que na família havia baixo nível de apoio e de conflitos.

Conglomerado 2 ( $n=22 ; 16,4 \%$ da amostra): formado por idosos independentes para atividades básicas e instrumentais de vida diária; com depressão e ansiedade; insatisfeitos com o nível de adaptação, companheirismo, desenvolvimento, afetividade e capacidade resolutiva da familia; que julgavam que sua família era disfuncional, nela predominando disfuncionalidade, baixo apoio, baixa coesão e alto nível de conflitos.
Conglomerado 3 ( $n=108 ; 80,6 \%$ da amostra): formado por idosos com independência para atividades básicas e instrumentais de vida diária; sem depressão e sem ansiedade; satisfeitos com a adaptação, o companheirismo, o desenvolvimento, a afetividade e a capacidade resolutiva da familia; que avaliavam com o boa a funcionalidade familiar; que relataram que em suas famílias predominavam alto nível de apoio e de coesão e baixo nível de conflitos.

$\mathrm{Na}$ Tabela 3, são mostradas as frequências das variáveis que compuseram os conglomerados, considerando-se a configuração familiar, as condições de saúde física e psicológica e o funcionamento familiar relatados pelos idosos. Observa-se que as variáveis que se associaram de maneira estatisticamente significativa foram as atividades básicas e instrumentais de vida diária, a ansiedade, a depressão, a adaptação, a satisfação com os relacionamentos familiares quanto ao companheirismo, ao desenvolvimento, à afetividade e a capacidade resolutiva e a percepção geral quanto à funcionalidade familiar, ao apoio, à coesão e ao conflito. Não se associaram significativamente ente si ou às demais variáveis: idade, sexo, arranjos de moradia, chefia familiar, contribuição para o sustento da família, envolvimento social, número de doenças, sinais e sintomas e hierarquia familiar, sinal de que se distribuíram de forma similar entre os grupos.

De modo geral, houve um percentual superior de idosos de 60 a 74 anos; nos grupos 2 e 3 e no grupo 1, as proporções de indivíduos pelas faixas etárias distribuíram-se igualmente. Nos três grupos, a maioria dos idosos eram mulheres, idosos que viviam com a família de um filho ou filha, com ou sem o cônjuge, chefes de família, e idosos com baixo envolvimento social. Nos grupos 1 e 2, observou-se maior percentual de

Tabela 1

Caracterização dos Conglomerados

\begin{tabular}{lccccc}
\hline Grupos & Frequência & $\begin{array}{c}\text { RMS }^{2} \text { (Desvio- } \\
\text { padrão) }\end{array}$ & $\begin{array}{c}\text { Distância máxima } \\
\text { observada do } \\
\text { centro }\end{array}$ & $\begin{array}{c}\text { Conglomerado } \\
\text { mais próximo }\end{array}$ & $\begin{array}{c}\text { Distâncias entre } \\
\text { os centróides dos } \\
\text { conglomerados }\end{array}$ \\
\hline 1 & 04 & 1,0596 & 4,9610 & 3 & 6,2728 \\
2 & 22 & 0,9686 & 6,4268 & 3 & 5,5416 \\
3 & 108 & 0,8673 & 6,4892 & 2 & 5,5416 \\
\hline
\end{tabular}

Nota. RMS² (Desvio padrão) = Índice RMSSTD (Root Mean Square Standard Deviation), cuja tradução pode ser raiz-quadrada do desvio padrão médio, é usado para calcular a homogeneidade dos agrupamentos. 
Tabela 2

Resultados da Análise de Conglomerados

\begin{tabular}{lccc}
\hline Variáveis & Desvio padrão & $\begin{array}{c}\text { Coeficiente de } \\
\text { determinação }\end{array}$ & ${\text { RSQ/(1-RSQ })^{\mathrm{b}}}^{\mathrm{a}}$ \\
\hline Sexo & 1,00211 & 0,010878 & 0,010998 \\
Idade & 1,00093 & 0,013209 & 0,013386 \\
Arranjos de moradia & 1,00473 & 0,005707 & 0,005740 \\
Chefia familiar & 1,00670 & 0,001788 & 0,001791 \\
Contribuição sustento da família & 1,00288 & 0,009361 & 0,009450 \\
$\mathrm{~N}^{\circ}$ doenças autorrelatadas & 0,98080 & 0,052493 & 0,055402 \\
$\mathrm{~N}^{\circ}$ sinais e sintomas autorrelatadas & 0,98803 & 0,038479 & 0,040018 \\
Envolvimento social & 1,00462 & 0,005914 & 0,005949 \\
ABVDs & 0,43197 & $\underline{0,816204}$ & 4,440819 \\
AIVDs & 0,95726 & 0,097426 & 0,107942 \\
Depressão & 0,97824 & 0,057438 & 0,060938 \\
Ansiedade & 0,93627 & 0,136572 & 0,158174 \\
Adaptação & 0,74643 & 0,822217 & 0,451218 \\
Companheirismo & 0,58594 & $\underline{0,661837}$ & 1,957154 \\
Desenvolvimento & 0,64207 & $\underline{0,593939}$ & 1,462687 \\
Afetividade & 0,56847 & $\underline{0,681701}$ & 2,141697 \\
Capacidade resolutiva & 0,65705 & $\underline{0,574774}$ & 1,351692 \\
Satisfação dinâmica familiar & 0,60232 & $\underline{0,642671}$ & 1,798540 \\
Apoio & 0,95950 & 0,093202 & 0,102781 \\
Coesão & 0,97152 & 0,070340 & 0,075662 \\
Conflito & 0,94961 & 0,111793 & 0,125863 \\
Hierarquia & 1,00469 & 0,005770 & 0,005804 \\
Total & 0,88911 & $\underline{0,221364}$ & 0,284297 \\
\hline
\end{tabular}

Nota. ${ }^{a}$ Coeficiente de determinação, $\mathrm{R}^{2}=$ (Quadrado do coeficiente de correlação), usado para calcular a dissimilaridade entre agrupamentos. broporção da variância dentro dos grupos $\left(\mathrm{R}^{2} /\left(1-\mathrm{R}^{2}\right)\right)$

contribuição total para o sustento familiar e, no grupo 3 , de contribuição parcial e total. Os idosos do grupo 1 e a maior parte dos do grupo 2 relataram ter três ou mais doenças, enquanto o grupo 3 relatou ter duas ou menos. A maioria dos idosos dos três grupos relatou ter três ou mais sinais e sintomas. Predominaram no grupo 1, idosos com alguma dependência em atividades básicas de vida diária e dependência total em atividades instrumentais de vida diária. Nos grupos 2 e 3, predominaram idosos independentes.

A depressão e ansiedade apresentaram-se de forma semelhante nos grupos 1 e 2 . No grupo 1, os idosos relataram satisfação com seus relacionamentos familiares mas avaliaram mais negativamente o apoio disponível. O grupo 2 apresentou insatisfação com os relacionamentos familiares, avaliação negativa do apoio, da coesão e do conflitos. A avaliação da hierarquia familiar apresentou-se de forma similar nos três grupos.

\section{Discussão}

O primeiro grupo foi composto por idosos que requerem ajuda para desempenhar atividades instrumentais e básicas de vida diária, uma situação de que pode afetar o equilíbrio da estrutura familiar, pois exige negociação de tarefas e flexibilidade. No Grupo 1, além da dependência funcional, havia depressão e ansiedade. O comprometimento funcional e a fragilidade na 
Tabela 3

Proporções de Idosos na Amostra e nos Conglomerados, Considerando-Se as Variáveis Sociodemográficas, as Condicões da Saúde Física e Mental e as Avaliações sobre a Funcionalidade Familiar

\begin{tabular}{|c|c|c|c|c|c|c|c|}
\hline \multirow[b]{2}{*}{ Variáveis } & \multirow[b]{2}{*}{ Categorias } & \multicolumn{2}{|c|}{ Amostra total } & \multicolumn{4}{|c|}{ Conglomerados } \\
\hline & & $\mathrm{n}$ & $\%$ & $\begin{array}{l}1 \\
\%\end{array}$ & $\begin{array}{l}2 \\
\%\end{array}$ & $\begin{array}{l}3 \\
\%\end{array}$ & $p^{*}$ \\
\hline \multirow[t]{2}{*}{ Idade } & 60-74 anos & 89 & 66,4 & 50,0 & 77,3 & 64,8 & 0,381 \\
\hline & $\geq 75$ anos & 45 & 33,6 & 50,0 & 22,7 & 35,2 & \\
\hline \multirow[t]{2}{*}{ Sexo } & Feminino & 104 & 77,6 & 100,0 & 72,7 & 77,8 & 0,598 \\
\hline & Masculino & 30 & 22,4 & 0 & 27,3 & 22,2 & \\
\hline \multirow{5}{*}{$\begin{array}{l}\text { Arranjos de } \\
\text { moradia }\end{array}$} & Sozinho & 24 & 17,9 & 25,0 & 27,3 & 15,8 & 0,705 \\
\hline & $\mathrm{C} /$ cônjuge/companheiro & 10 & 7,5 & 0 & 4,5 & 8,3 & \\
\hline & C/ cônjuge e descendentes & 32 & 23,9 & 25,0 & 18,2 & 25,0 & \\
\hline & Com descendentes & 55 & 41,0 & 50,0 & 31,8 & 42,6 & \\
\hline & Outros & 13 & 9,7 & 0 & 18,2 & 8,3 & \\
\hline \multirow[t]{2}{*}{ Chefia familiar } & $\operatorname{Sim}$ & 97 & 72,4 & 75,0 & 68,2 & 73,2 & 0,835 \\
\hline & Não & 37 & 27,6 & 25,0 & 31,8 & 26,8 & \\
\hline \multirow{3}{*}{$\begin{array}{l}\text { Contribuição para } \\
\text { o sustento família }\end{array}$} & Total & 66 & 49,3 & 75,0 & 54,5 & 47,2 & 0,727 \\
\hline & Parcial & 59 & 44,0 & 25,0 & 36,4 & 46,3 & \\
\hline & Nenhuma & 09 & 6,7 & 0 & 9,1 & 6,5 & \\
\hline \multirow{2}{*}{$\begin{array}{l}\text { Envolvimento } \\
\text { social }\end{array}$} & Menor & 88 & 65,7 & 75,0 & 72,7 & 63,9 & 0,734 \\
\hline & Maior & 46 & 34,3 & 25,0 & 27,3 & 36,1 & \\
\hline \multirow{3}{*}{$\begin{array}{l}\mathrm{N}^{\circ} \text { doenças } \\
\text { autorrelatadas }\end{array}$} & Nenhuma & 18 & 13,4 & 0 & 13,6 & 13,9 & 0,051 \\
\hline & $1-2$ & 70 & 52,3 & 0 & 40,9 & 56,5 & \\
\hline & $\geq 3$ & 46 & 34,3 & 100,0 & 45,5 & 29,6 & \\
\hline \multirow{3}{*}{$\begin{array}{l}\mathrm{N}^{\circ} \text { sinais } \\
\text { e sintomas } \\
\text { autorrelatadas }\end{array}$} & Nenhum & 12 & 9,0 & 0 & 0 & 11,1 & 0,295 \\
\hline & $1-2$ & 42 & 31,3 & 25,0 & 22,7 & 33,3 & \\
\hline & $\geq 3$ & 80 & 59,7 & 75,0 & 77,3 & 55,6 & \\
\hline \multirow[t]{3}{*}{ ABVDs } & Independentes & 129 & 96,3 & 0 & 25,4 & 100,0 & $\leq 0,001$ \\
\hline & Com dependência parcial & 03 & 2,2 & $\underline{50,0}$ & 4,6 & 0 & \\
\hline & Dependentes & 02 & 1,5 & $\underline{50,0}$ & 0 & 0 & \\
\hline \multirow[t]{3}{*}{ AIVDs } & Com dependência total & 08 & 6,0 & $\underline{75,0}$ & 13,6 & 1,8 & $<0,001$ \\
\hline & Com dependência parcial & 48 & 35,8 & 25,0 & 40,9 & 35,2 & \\
\hline & Independentes & 78 & 58,2 & 0 & $\underline{45,5}$ & $\underline{63,0}$ & \\
\hline \multirow[t]{2}{*}{ Depressão } & $\operatorname{Sim}$ & 23 & 17,2 & $\underline{50,0}$ & $\underline{31,8}$ & 13,0 & 0,018 \\
\hline & Não & 111 & 82,8 & 50,0 & 68,2 & $\underline{87,0}$ & \\
\hline \multirow[t]{2}{*}{ Ansiedade } & $\operatorname{Sim}$ & 31 & 23,1 & $\underline{75,0}$ & $\underline{50,0}$ & 15,7 & $<0,001$ \\
\hline & Não & 103 & 76,9 & 25,0 & 50,0 & $\underline{84,3}$ & \\
\hline
\end{tabular}


Tabela 3

Proporções de Idosos na Amostra e nos Conglomerados, Considerando-Se as Variáveis Sociodemográficas, as Condicões da Saúde Física e Mental e as Avaliaçoes sobre a Funcionalidade Familiar (Continuação)

\begin{tabular}{|c|c|c|c|c|c|c|c|}
\hline \multirow[b]{2}{*}{ Variáveis } & \multirow[b]{2}{*}{ Categorias } & \multicolumn{2}{|c|}{ Amostra total } & \multicolumn{4}{|c|}{ Conglomerados } \\
\hline & & $\mathrm{n}$ & $\%$ & $\begin{array}{l}1 \\
\%\end{array}$ & $\begin{array}{l}2 \\
\%\end{array}$ & $\begin{array}{l}3 \\
\%\end{array}$ & $p^{*}$ \\
\hline \multirow[t]{2}{*}{ Adaptação } & Insatisfeitos & 25 & 18,7 & 25,0 & 77,3 & 6,5 & $<0,001$ \\
\hline & Satisfeitos & 109 & 81,3 & $\underline{75,0}$ & 22,7 & $\underline{93,5}$ & \\
\hline \multirow[t]{2}{*}{ Companheirismo } & Insatisfeitos & 23 & 17,2 & 0 & $\underline{86,4}$ & 3,7 & $<0,001$ \\
\hline & Satisfeitos & 111 & 82,8 & $\underline{100,0}$ & 13,6 & $\underline{96,3}$ & \\
\hline \multirow[t]{2}{*}{ Desenvolvimento } & Insatisfeitos & 25 & 18,7 & 0 & $\underline{86,4}$ & 5,6 & $<0,001$ \\
\hline & Satisfeitos & 109 & 81,3 & $\underline{100,0}$ & 13,6 & $\underline{94,4}$ & \\
\hline \multirow[t]{2}{*}{ Afetividade } & Insatisfeitos & 26 & 19,4 & 50,0 & $\underline{90,9}$ & 3,7 & $<0,001$ \\
\hline & Satisfeitos & 108 & 80,6 & 50,0 & 9,1 & $\underline{96,3}$ & \\
\hline \multirow{2}{*}{$\begin{array}{l}\text { Capacidade } \\
\text { resolutiva }\end{array}$} & Insatisfeitos & 21 & 15,7 & 25,0 & 77,3 & 2,8 & $<0,001$ \\
\hline & Satisfeitos & 113 & 84,3 & $\underline{75,0}$ & 22,7 & $\underline{97,2}$ & \\
\hline \multirow{2}{*}{$\begin{array}{l}\text { Satisfação com a } \\
\text { dinâmica familiar }\end{array}$} & Disfunção familiar & 19 & 14,2 & 0 & $\underline{77,3}$ & 1,8 & $<0,001$ \\
\hline & Boa funcionalidade & 115 & 85,8 & $\underline{100,0}$ & 22,7 & $\underline{98,2}$ & \\
\hline \multirow[t]{2}{*}{ Apoio } & Menor & 78 & 58,2 & $\underline{75,0}$ & $\underline{90,9}$ & 50,9 & $<0,001$ \\
\hline & Maior & 56 & 41,8 & $\overrightarrow{25,0}$ & $\overrightarrow{9,1}$ & $\underline{49,1}$ & \\
\hline \multirow[t]{2}{*}{ Coesão } & Menor & 76 & 56,7 & 50,0 & 86,4 & $\underline{50,9}$ & $\underline{0,004}$ \\
\hline & Maior & 58 & 43,3 & 50,0 & 13,6 & $\underline{49,1}$ & \\
\hline \multirow[t]{2}{*}{ Conflito } & Menor & 68 & 50,7 & 75,0 & 13,6 & $\underline{57,4}$ & $\leq 0,001$ \\
\hline & Maior & 66 & 49,3 & 25,0 & 86,4 & $\underline{42,6}$ & \\
\hline \multirow[t]{2}{*}{ Hierarquia } & Menor & 72 & 53,7 & 50,0 & 45,4 & $\underline{55,6}$ & $\underline{0,702}$ \\
\hline & Maior & 62 & 46,3 & 50,0 & 54,6 & $\underline{44,4}$ & \\
\hline
\end{tabular}

velhice estão associadas a essas condições psicológicas (Ní Mhaol'ain et al., 2012) e a níveis mais baixos de bem-estar, possivelmente em decorrência de uma crise de identidade refletida em resposta psicológica mal adaptativa a um evento de vida desafiador e às perdas em saúde (Andrew, Fisk, \& Rockwood, 2012). Para os idosos nessas condições, a presença de outras pessoas que valorizem sua história e que validem os rituais diários que ancoram sua experiência é vital para sua qualidade de vida (Nicholson, Meyer, Flatley, Holman, \& Lowton, 2012).

O estresse pode associar-se a prejuízo à funcionalidade familiar, até que a família se reorganize em um novo modo de funcionamento. Indícios desse processo de reorganização familiar pode ser observada na avaliação da família, que no grupo 1 obteve resultados diferentes quando feita de forma geral pela satisfação e quando avaliada por domínios mais específicos que exploraram mais de perto aspectos comportamentais, cognitivos e afetivos. Os idosos do primeiro grupo mostraram satisfação com os relacionamentos familiares concernentes à capacidade de adaptação, ao companheirismo, ao desenvolvimento e à capacidade resolutiva. Por outro lado, perceberam menor apoio familiar, indicando uma ponderação mais negativa da reciprocidade nas relações, mesmo tendo mostrado satisfação com 
as relações familiares. É possível que os idosos tenham sido realistas ao avaliar suas circunstâncias, demandas situacionais e estressores e reconhecendo que precisam de assistência, mas pode ser que a família não seja capaz de responder a todas as necessidades que derivam dessa condição. No entanto, não se sabe como essas famílias funcionavam previamente e em que medida essas características refletem ou não refletem um padrão de funcionamento anteriormente estabelecido.

Além disso, é provável que o fato do primeiro grupo ter apresentado satisfação com os relacionamentos familiares, mas percepção de menor apoio, tenha a ver com a questão de que o suporte de familiares a idosos dependentes é seletivo e as transferências de apoio não se equivalem em diversos contextos. Isso significa que eles podem estar satisfeitos com o apoio recebido de alguns membros, mas não de todos. Nem todos assumem os cuidados ou estão sensibilizados para com as necessidades dos idosos. Ainda, pode ser que os idosos estejam sendo cuidados, mas não da forma como gostariam ou esperam. Há ainda a questão da transferência dos recursos do idoso na família, que pode deixar alguns filhos ou parentes insatisfeitos. Essas situações podem resultar em problemas na percepção de apoio na família como um todo (Silverstein, 2006). Por outro lado, é preciso destacar que os idosos tendem a avaliar positivamente a sua família porque isso protege seu autoconceito e sua autoestima. Avaliar negativamente a família pode significar insucesso pessoal e ameaça ao senso de autorrealização.

Percepção negativa de suporte social está relacionada à depressão em vários estudos, no entanto pouco se sabe sobre quais aspectos do suporte são mais afetados e de que maneira (Marroquín, 2011). Na presença de dependência, as expectativas socialmente normativas com relação ao cuidado de idosos pelos familiares podem apresentar diferentes efeitos sobre a satisfação e sobre os afetos positivos. A percepção de que o suporte é positivo e suficiente parece ter efeitos benéficos sobre as emoções quando quem o recebe percebe equilíbrio nas trocas. Esse efeito pode estar relacionado aos custos do suporte quando é socialmente visível, tais como o sentimento de dívida, de incompetência ou de baixo senso de autoeficácia (Bolger \& Amarel, 2007).

A percepção de pouco conflito familiar foi predominante no primeiro grupo, o que pode indicar que essa família esteja se ajustando às novas necessidades do idoso, o que afeta a qualidade positiva das relações, mas não muda a sua valência negativa. Sugere a presença de uma forma passiva de enfrentamento, como, por exemplo, distanciar-se de situações conflituosas a fim de preservar recursos físicos, sociais e psicológicos, especialmente porque apresentam comprometimento funcional.

Embora independente funcionalmente, o segundo grupo mostrou pior condição psicológica, insatisfação com os relacionamentos familiares (em todos os domínios), e uma percepção de menor apoio, menor coesão e maior conflito. As relações interpessoais influenciam a regulação emocional e essa relação é bidirecional. A depressão é uma experiência emocional caracterizada por baixo afeto positivo e alto afeto negativo, por uma visão negativa de si mesmo, do mundo e do futuro e por uma regulação mal adaptativa da emoção. Esses fatores estão relacionados com baixo suporte social, menor aproximação a outras pessoas e menor satisfação social. A habilidade de regular e manejar as emoções de forma adaptativa está associada com a satisfação com os relacionamentos sociais, com mais interações sociais positivas e com menos interações negativas (Marroquín, 2011).

Não se sabe se a disfunção familiar contribuiu para mais sintomas depressivos ou o contrário, mas se sabe que os familiares podem perpetuar os sintomas psiquiátricos do idoso bem como o idoso pode intensificar seus sintomas e a percepção de disfuncionalidade familiar em decorrência da desregulação emocional própria da depressão (Blazer, 2003). O conflito familiar está associado a transtornos de ansiedade (Priest \& Denton, 2012), e afeta significativamente o funcionamento familiar quando a coesão é baixa. Interações negativas e conflituosas podem indicar uma dificuldade em equilibrar as necessidades individuais e as da unidade familiar.

A percepção de mais conflito familiar observado no segundo grupo pode ter relação com as tensões advindas da luta por autonomia dos idosos. A autonomia é um valor cultural importante e um anseio de pessoas de todas as idades (Lowenstein, 2007). A perda de independência física, cognitiva e financeira, com a consequente necessidade de aceitação de ajuda e a mudança de papeis familiares é um grande desafio na velhice. A família pode criar um ambiente adverso à expressão de liberdade e autonomia de seu membro familiar mais velho (Reis et al., 2011). Conflitos podem emergir nos relacionamentos familiares com relação ao controle e ao manejo de recursos, à tomada de decisões, às diferenças em valores e expectativas quanto ao cumprimento de papéis ou devido a uma percepção inapropriada de dependência de uma geração em relação a outra (Piercy, 2010). 
Os idosos do segundo grupo são independentes, mas com piores condições psicológicas, não se sabe se antecedentes ou consequentes às avaliações sobre as relações familiares. De todo modo, a necessidade de suporte social é latente. A troca positiva de suporte social requer uma comunicação clara da necessidade bem como a habilidade do provedor de suporte corresponder à necessidade. Os indivíduos podem ter expectativas irrealistas quanto a comportamentos de suporte por parte de familiares. A má comunicação pode interferir tanto na apropriada provisão de suporte quanto na percepção da adequação do suporte recebido. O suporte familiar bem intencionado, mas equivocado, pode resultar em superproteção ou em aconselhamento negativo e mal informado (Fingerman \& Birditt, 2011).

Nota-se que a coesão e a satisfação com a afetividade dizem respeito à proximidade emocional no contexto familiar e foram avaliados negativamente no grupo 2. A qualidade emocional das ligações familiares influencia o bem-estar emocional e, na velhice, a proximidade emocional (percepção de afetuosidade física, verbal e emocional) é o principal mecanismo psicológico de regulação dos relacionamentos sociais e do esforço empreendido na manutenção desses relacionamentos (Lang et al, 2013). Segundo Priest e Denton (2012), em famílias latinas, a coesão está associada com menor estresse e com menos problemas de ansiedade. Inclui priorizar as obrigações familiares, honrar a família e usá-la como referência para a definição do self.

No terceiro grupo, que equivale a maior parte da amostra, associaram-se independência funcional, boa saúde psicológica, percepção de boa funcionalidade familiar, satisfação com os relacionamentos familiares e avaliações positivas do clima familiar. As avaliações dos idosos desse grupo podem ter sido afetadas pela independência e pela ausência de sintomas depressivos e de ansiedade, pelo alto grau de adaptabilidade das suas famílias ou, provavelmente, por ambos os fatores. Essa adaptabilidade familiar reflete-se em maior resiliência, que, ao mesmo tempo, possibilita aos idosos um ajustamento positivo frente aos desafios da velhice e o atendimento mais adequado das necessidades dos membros da família (Marin \& Huber, 2011).

Os padrões de expressão emocional positiva e negativa contribuem para a socialização da emoção e fazem parte de um conjunto de regras estabelecidas para sua expressão na família. Relações familiares positivas e um ambiente coeso dão o tom de aceitação para a experiência emocional e para a expressão de necessidades (Fosco \& Grych, 2012). Quando coerente com o estado interno das pessoas, a expressão de emoções positivas leva a níveis mais altos de funcionamento psicológico e ao aumento da conectividade social (Mauss et al., 2011).

O terceiro grupo caracterizou as próprias famílias como funcionais, sugerindo que elas têm boa capacidade de absorver e de lidar com as situações de crise de forma realista e adequada e de cumprir e harmonizar suas funções essenciais. Suas avaliações indicam que são robustos o vínculo emocional e o compartilhamento de afeto e apoio nas famílias. O funcionamento psicológico positivo dos idosos observado nesse grupo deve ter relações recíprocas com essas características das famílias.

\section{Considerações Finais}

Como limitação do estudo, pode-se citar a ausência de informações sobre a percepção de outros membros da família, as quais pudessem ampliar o entendimento do funcionamento familiar como um todo. Sugere-se que futuros estudos incluam métodos mais refinados de análise de dados, delineamentos longitudinais e abordagens multirreferenciada e multinivel envolvendo tanto o idoso quanto outros familiares. Faz-se necessária, a construção de instrumentos brasileiros refinados e sensíveis às peculiaridades culturais.

Os dados sugerem que as condições psicológicas afetam mais a percepção da qualidade das relações familiares dos idosos independentes (grupos 2 e 3 ) do que a saúde física, tendo em vista que o grupo 2 relata piores condições psicológicas e piores relações familiares e o grupo 3 descreve melhores condições psicológicas e melhores relações familiares. Idosos funcionalmente independentes podem perceber o funcionamento familiar como saudável ou não. Não se conhecem as relações de causa e efeito entre depressão e ansiedade e pior avaliação familiar, mas se pode dizer que estão associadas, assim como estão associadas à desregulação emocional própria dessas condições psicológicas, os padrões de interação familiar e a capacidade das famílias de atender às demandas desse idoso. Ao mesmo tempo, as famílias com idosos dependentes podem ter dificuldades de ajustamento à dependência deles. Isso envolve reorganização e modificação de padrões construídos ao longo do tempo e, por vezes, desfechos negativos de saúde psicológica. Apesar do pequeno número de idosos dependentes e com depressão e ansiedade na 
amostra, esses grupos ofereceram pistas importantes a serem seguidas em busca de entendimento de como vivem pessoas nessas condições.

\section{Referências}

Almeida, O. P. \& Almeida, S. A. (1999). Short version of the geriatric depression scale: A study of their validity for the diagnosis of major depressive episode according to ICD-10 and DSM-IV. International Journal of Geriatric Psychiatric, 14(10), 858-865.

Andrew, M. K., Fisk, J. D., \& Rockwood, K. (2012). Psychological well-being in relation to frailty: a frailty identity crisis? International Psychogeriatrics, 24(8), 1347-1353. doi: 10.1017/S1041610212000269

Batistoni, S. S. T., Neri, A. L., Tomomitsu, M. R. S. V., Vieira, L. A. M., Oliveira, D., Cabral, B. E., \& Araújo, L. F. (2013). Arranjos domiciliares, suporte social, expectativa de cuidado e fragilidade. In A. L. Neri (Ed), Fragilidade e qualidade de vida na velhice (pp. 267-282). Campinas, SP: Alínea.

Blazer, D. (2003). Depressão em idosos. São Paulo: Andrei.

Bolger, N., \& Amarel, D. (2007). Effects of social support visibility on adjustment to stress: experimental evidence. Journal of Personality and Social Psychology, 92(3), 458-475. doi: 10.1037/0022-3514.92.3.458

Brucki, S. M. D., Nitrini, R., Caramelli, P., Bertolucci, P. H. F., \& Okamoto, I. H. (2003). Sugestões para o uso do mini-exame do estado mental no Brasil. Arquivos de Neuropsiquiatria, 61(3-B), 777-781.

Camarano, A. A., \& Kanso, S. (2010). Como as famílias brasileiras estão lidando com idosos que demandam cuidados e quais as perspectivas futuras? A visão mostrada pelas PNADS. Em A. A. Camarano (Ed.), Cuidados de longa duração para a população idosa: um novo risco social a ser assumido? (pp. 93-122). Rio de janeiro: IPEA.

Carl, J. R., Soskin, D. P., Kerns, C., \& Barlow, D. H. (2013). Positive emotion regulation in emotional disorders: A theoretical review. Clinical Psychology Review, 33(3), 343-360. doi: 10.1016/j.cpr.2013.01.003

Cunha, J. A. (2001). Manual da versão em português das Escalas Beck. São Paulo: Casa do Psicólogo.

Fingerman, K. L., \& Birditt, K. S. (2011). Relationships between adults and their aging parents. In K. W.
Schaie \& S. L. Willis (Eds.), Handbook of Psychology of aging (pp. 219-229). San Diego: Elsevier.

Fosco, G. M., \& Grych, J. H. (2012). Capturing the family context of emotion regulation: a family systems model comparison approach. Journal of Family Issues, 34(4), 557-578. doi: 10.1177/0192513X12445889

Hyde, L. W., Gorka, A., Manuck, S. B., \& Hariri A. R. (2011). Perceived social support moderates the link between threat-related amygdale reactivity and trait anxiety. Neuropsychologia, 49, 651-656. doi: 10.1016/j.neuropsychologia.2010.08.025

Kaufman, L., \& Rousseeuw, P. J. (1990). Finding groups in data: An introduction to Cluster Analysis. New York: John Wiley \& Sons.

Lang, F. R., Wagner, J., Wrzus, C., \& Neyer, F. J. (2013). Personal effort in social relationships across adulthood. Psychology and Aging, 28(2), 529-539. doi: 10.1037/a0032221

Lino, V. T. S., Pereira, S. E. M., Camacho, L. A. B., Ribeiro Filho, S. T., \& Buksman, S. (2008). Adaptação transcultural da Escala de Independência em Atividades de Vida Diária (Escala de Katz). Cadernos de Saúde Pública, 24(1), 103-112.

Lowenstein, A. (2007). Solidarity-conflict and ambivalence: Testing two conceptual frameworks and their impact on quality of life for older family members. Journal of Gerontology: Social Sciences, 62(2), 100-107.

Marin, M., \& Huber, C. H. (2011). Mexican American elderly: Self-reported anxiety and the mediating influence of family protective factors. The Family Journal, 19(1), 63-72. doi:10.1177/1066480710387394

Marroquín, B. (2011). Interpersonal emotion regulation as a mechanism of social support in depression. Clinical Psychology Reviem, 31, 1276-1290. doi: 10.1016/j.cpr.2011.09.005

Mauss, I. B., Shallcross, A. J., Troy, A. S., John, O. P., Ferrer, E., Wilhelm, F. H., \& Gross, J. J. (2011). Don't hide your happiness! Positive emotion dissociation, social connectedness, and psychological functioning. Journal of Personality and Social Psychology, 100(4), 738-48. doi: 10.1037/a0022410

Neri, A. L.,\& Guariento, M. E. (2011). Fragilidade, saúde e bem-estar em idosos: dados do Estudo FIBR A Campinas. Campinas, SP: Alínea. 
Neri, A. L., Yassuda, M. S., Araújo, L. F., Eulálio, M. C., Cabral, B. E., ... Siqueira, M. E. C. (2013). Metodologia e perfil sociodemográfico, cognitivo e de fragilidade de idosos comunitários de sete cidades brasileiras: Estudo FIBRA. Cadernos de Saúde Pública, 29(4), 778-792.

Nicholson, C., Meyer, J., Flatley, M., Holman, C., \& Lowton, K. (2012). Living on the margin: Understanding the experience of living and dying with frailty in old age. Social Science \& Medicine, 75(8), 1426-1432.

Ní Mhaol'ain, A. M., Gallagher, D., O Connell, H., Chin, A. V., Bruce, I., Hamilton, F., Teehee, E., Coen, R., Coakley, D., Cunningham, C., Walsh, J. B., \& Lawlor, B. A. (2012). Subjective well-being amongst community-dwelling elders: what determines satisfaction with life? Findings from the Dublin Healthy Aging Study. International Psychogeriatrics, 24(2), 316-323, 2012. doi: 10.1017/S1041610211001360

Paiva, A. T. G., Bessa, M. E. P., Moraes, G. L. A., Silva, M. J., Oliveira, R. D. P., \& Soares, A. M. G. (2011). Avaliação da funcionalidade de famílias com idosos. Cogitare Enfermagem, 16(1), 22-28.

Pavarini, S. C. L., Tonon, F. L., Silva, J. M. C., Mendiondo, M. Z., Barham, E. J., \& Filizola, C. L. A. (2006). Quem irá empurrar minha cadeira de rodas? A escolha do cuidador familiar do idoso. Revista Eletrônica de Enfermagem, 8(3), 326-335.

Pereira, J. C. R. (1999). Análise de dados qualitativos: Estratégias metodológicas para as ciências da saúde, bumanas e sociais. São Paulo: EdUSP-Fapesp.

Piercy, K. W. (2010). Understanding family dynamics. In K. W. Piercy (Ed.), Working with aging families (pp. 41-72). New York: W.W. Norton \& Company.

Priest, J. B., \& Denton, W. (2012). Anxiety Disorders and Latinos: The Role of Family Cohesion and Family Discord. Hispanic Journal of Behavioral Sciences, 34(4), 557-575. doi:10.1177/0739986312459258

Reis, L. A., Torres, G. V., Xavier, T. T., Silva, R. A. R., Costa, I. K. F,. \& Mendes, F. R. P. (2011). Percepção do suporte familiar em idosos de baixa renda e fatores associados. Texto \& Contexto Enfermagem, 20, 52-58.

Resende, M. C., Turra, D. D., Alves, F., Pereira, F. B., Santos, S. A. P., \& Trevisan, V. C. (2008). Cuidar de idosos com Alzheimer: Influências sociais, físicas e psicológicas envolvidas nesta tarefa. Revista Brasileira de Ciências do Envelhecimento Humano, 5(1), 19-31. doi: 10.5935/0034-7167.20140030

Rodríguez-Sánchez, E., Pérez-Penaranda, A., Losada-Baltar,A., Pérez-Arechaederra,D., Gómez-Marcos, M. A., Patino-Alonso, M. C., \& Garcia-Ortiz, L. (2011). Relationships between quality of life and family function in caregiver. BMC Family Practice, 12(1), 12-19. doi: 10.1186/1471-2296-12-19

Santos, A. A., Pavarini, S. C. L., \& Barham, E. J. (2011). Percepção de idosos pobres com alterações cognitivas sobre funcionalidade familiar. Texto \& Contexto de Enfermagem, 20(1),102-110.

Santos, R. L., \& Virtuoso-Júnior, J. S. (2008). Confiabilidade da versão brasileira da escala de atividades instrumentais de vida diária. Revista Brasileira de Promoção da Saúde, 21(4), 290-296.

Silva, H., Rabelo, D. F. \& Queroz, N. C. (2010). Qualidade de vida, percepção da dinâmica familiar e do suporte social em idosos. Pensando Famílias, 14(2), 137-150.

Silverstein, M. (2006). Intergenerational family transfers in social context. Em R. H. Binstock \& L. George (Eds), Handbook of Aging and the Social Sciences (pp. 165-180). New York: Academic Press.

Silverstein, M., \& Giarusso, R. (2010). Aging and family life: A decade review. Journal of Marriage and family, 72, 1039-1058. doi: 10.1111/j.1741-3737.2010.00749.x

Smilkstein G. (1978). The Family APGAR: A proposal for family function test and its use by physicians. Journal of Family Practice, 6(6),1231-39.

Teodoro, M. L. M., Allgayer, M., \& Land, B. (2009). Desenvolvimento e validade fatorial do Inventário do Clima Familiar (ICF) para adolescentes. Psicologia: Teoria e Prática, 11(3), 27-39.

Torres, G. V., Reis, L. A., Reis, L. A., \& Fernandes, M. H. (2009). Qualidade de vida e fatores associados em idosos dependentes em uma cidade do interior do Nordeste. Jornal Brasileiro de Psiquiatria, 58(1), 39-44.

Torres, G. V., Reis, L. A., Reis, L. A., Fernandes, M. H., \& Xavier, T. T. (2010). Relação entre funcionalidade familiar e capacidade funcional de idosos dependentes no município de Jequié (BA). Revista Baiana de Saúde Pública, 34(1), 19-30. 
Vink, D., Aartsen, M. J., \& Shoevers, R. A. (2008). Risk factors for anxiety and depression in the elderly: a review. Journal of Affective Disorders, 106(1-2), 29-44.

Werner-Seidler, A., Banks, R., Dunn, B. D., \& Moulds, M. L. (2013). An investigation of the relationship between positive affect regulation and Depression. Behaviour Research and Therapy, 51(1), 46-56. doi: 10.1016/j.brat.2012.11.001

$1^{a}$ Reformulação 16/06/2015

$2^{\mathrm{a}}$ Reformulação 17/08/2015

Aceito 25/09/2015

Nota das autoras:

Dóris Firmino Rabelo é psicóloga, mestre em Gerontologia, doutora em Educação e docente do Centro de Ciências da Saúde da Universidade Federal do Recôncavo da Bahia.

E-mail: drisrabelo@yahoo.com.br

Anita Liberalesso Neri é psicóloga, professora titular no Departamento de Psicologia Educacional e docente do Programa de Pós-graduação da Faculdade de Ciências Médicas da UNICAMP.

E-mail: anitalbn@uol.com.br

Contato com as autoras:

Centro de Ciências da Saúde - UFRB

Av. Carlos Amaral, 1015 - Cajueiro

CEP: 44570-000

Santo Antônio de Jesus-Bahia, Brasil 